\title{
Detection of Grid-Shaped Mosaic Regions through Geometric Filtering from Social Video Data
}

\author{
Seok-Woo Jang ${ }^{1}$, Sung-Youn Cho ${ }^{2 *}$ \\ ${ }^{1,2}$ Department of Software, Anyang University, Anyang 14028, Republic of Korea \\ ${ }^{1}$ swjang7285@gmail.comk, ${ }^{2}$ scho@anyang.ac.kr
}

\begin{abstract}
This paper proposes an algorithm that accurately detects the grid-shaped mosaic region used to cover certain regions of video data based on edge projection. The proposed algorithm first detects Canny edges from the image and detects candidate regions of the mosaic using horizontal and vertical line edge projection. The actual mosaic regions are then finally detected by filtering candidate regions of the mosaic using geometric features. Experimental results show that the proposed algorithm detects the area corresponding to the mosaic block more accurately than the other detection methods from various input images.
\end{abstract}

Keywords: image analysis, feature extraction, mosaic detection, filtering, rule generation

\section{Introduction}

In general, video contains useful data, but may contain information that users do not want exposed to others. For example, the Google street view [1], which can be viewed frely by the general public through an Internet browser to search the map, may show the person's face, license plate, etc., which is one of the specific personal information. In addition, if you search the blog on the Internet, the uploaded photos may contain parts of other people's faces or exposed bodies that users do not want to be photographed. Therefore, many mosaics are used recently to hide these areas[2].

In application fields related to the detection of adult images [3], an area of a mosaic block is detected to effectively determine whether the image includes information that is not desired by the user, such as a specific part of the exposed body [4].

Existing research related to the field of automatically detecting mosaic regions from such digital image data can be found in the related literature. In[5], an edge is detected from an input image and a candidate region of a mosaic is generated based on the detected edge. Subsequently, the actual mosaic area is finally selected through filtering using geometric features. In[6], fuzzy C-means clustering algorithm is used to detect mosaic blocks accurately. In [7], edges are detected from an image, and candidate regions of a mosaic are detected using template matching. Finally, the actual mosaic area is selected using a support vector machine. In addition to these methods, various other approaches for detecting mosaics have been tried [8].

The existing methods described above are capable of detect the mosaic regions to some extent, but the accuracy is not high. To this day, researches on algorithms for generating and detecting mosaics have not been actively conducted. Therefore, in this paper, we propose a new algorithm to effectively detect grid-type mosaics based on edge projection. In this paper, we

Article history:

Received (August 11, 2019), Review Result (September 29, 2019), Accepted (October 26, 2019) 
mainly use mosaics to cover parts of the body in harmful images. In general, the mosaics used in the harmful image may have different sizes depending on the area to be masked, but a gridtype mosaic block, which is a relatively simple form, is mainly used rather than a complicated form. Although harmful images using blurring or virtual object insertion can be found, mosaic blocks tend to be used in many adult images.

Figure 1 shows the overall flow chart of the proposed mosaic detection algorithm. As shown in Figure 1, the proposed algorithm first extracts edge features from the input image and then detects candidate regions of the mosaic based on horizontal and vertical line edge projection. Subsequently, the final actual mosaic regions are detected by removing the non-mosaic regions through robust filtering of the candidate mosaic regions.

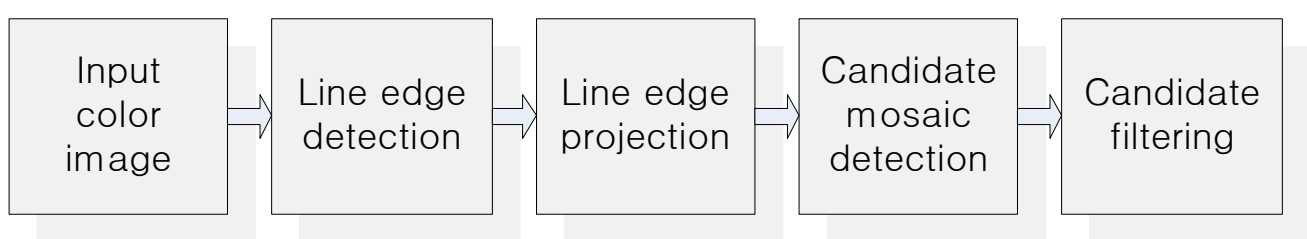

Figure 1. Overall flow of the proposed algorithm

\section{Extraction of candidate mosaics}

In this paper, we extract the Canny edge $\mathrm{E}(\mathrm{x}, \mathrm{y})$ from the input image [9]. In the color image, the edge is one of the important features for detecting the mosaic, so it is very important to accurately extract the edge image, which is the first step. In general, Canny edge is one of the most widely used edge detectors in image processing and computer vision because of its relatively high accuracy in finding contours.

The proposed system detects edges connected continuously over $\mathrm{TH}_{\text {line }}$ pixels in the horizontal direction from the extracted Canny edge, which is called horizontal line edge $\mathrm{LE}_{\mathrm{h}}$ $(\mathrm{x}, \mathrm{y})$ in this paper. In addition, edges continuously connected to a $\mathrm{TH}_{\text {line }}$ pixels or more in the vertical direction are detected and named as vertical line edge $\mathrm{LE}_{\mathrm{v}}(\mathrm{x}, \mathrm{y})$.

Then, as shown (1), the vertical line edge $\operatorname{LE}_{\mathrm{v}}(\mathrm{x}, \mathrm{y})$ is projected in the direction of the $\mathrm{x}$ axis. Subsequently, a set of $\mathrm{x}$ coordinates in which Proj(x), the number of pixels accumulated through projection, is equal to or greater than $\mathrm{TH}_{\text {accum }}$ is obtained and set to $\mathrm{X}_{\text {accum }}$.

$$
\begin{aligned}
& \operatorname{Proj}(x)=\sum_{x=0}^{W} L E_{v}(x, y) \\
& \operatorname{Proj}(y)=\sum_{y=0}^{H} L E_{h}(x, y)
\end{aligned}
$$

Similarly, the horizontal line edge $\operatorname{LE}_{\mathrm{h}}(\mathrm{x}, \mathrm{y})$ is projected in the direction of the $\mathrm{y}$ axis, and a set of y coordinates in which $\operatorname{Proj}(\mathrm{y})$, which is the number of pixels accumulated through the projection, is equal to or greater than $\mathrm{TH}_{\text {accum }}$ is obtained. In (1) and (2), $\mathrm{W}$ and $\mathrm{H}$ represent the width and height of the input image.

We compute the difference $X_{\text {diff }}$ between the adjacent $x$ coordinates in the set $X_{\text {accum }}$, and the difference $Y_{\text {diff }}$ between adjacent y coordinates in the set $Y_{\text {accum }}$. Subsequently, of $X_{\text {diff }}$ and $Y_{\text {diff, }}$, the value having the highest frequency and the value having the lowest frequency are selected 
and determined as $\mathrm{N}$ and $\mathrm{M}$ of the mosaic blocks having a size equivalent to the $\mathrm{N} \times \mathrm{M}$ pixel size. We select $\mathrm{x}$ coordinates where the difference between adjacent $\mathrm{x}$ coordinates in the set $\mathrm{X}_{\text {accum }}$ is $\mathrm{N}$ and $\mathrm{y}$ coordinates where the difference between adjacent $\mathrm{y}$ coordinates in the set $\mathrm{Y}_{\text {accum }}$ is $\mathrm{M}$, and they are set to $\mathrm{X}_{\text {mosaic }}$ and $\mathrm{Y}_{\text {mosaic }}$, respectively.

The minimum enclosing rectangle regarding the domain including all the locations (x,y) having a value included in $\mathrm{X}_{\text {mosaic }}$ as the input image's $\mathrm{x}$ coordinate and a value included in $\mathrm{Y}_{\text {mosaic }}$ as the input image's y coordinate are selected, and the defined MER is selected as the candidate mosaic block to be detected.

\section{Detection of real mosaic}

In this paper, geometric features are used to remove candidate regions that are considered to be non-mosaic regions among the candidate regions of the grid-type mosaic detected in the previous step. Geometric features used to remove candidate regions are defined by using the size features, aspect ratio features, and compactness features of mosaic candidate regions as shown in (3).

$$
\begin{aligned}
& \operatorname{size}\left(R_{i}\right)=\frac{\operatorname{Num}\left(R_{i}\right)}{W \times H} \\
& \operatorname{aspect}\left(R_{i}\right)=\frac{\operatorname{MER}_{h}\left(R_{i}\right)}{\operatorname{MER}_{w}\left(R_{i}\right)} \\
& \operatorname{compact}\left(R_{i}\right)=\frac{\operatorname{Num}\left(R_{i}\right)}{\operatorname{MER}_{w}\left(R_{i}\right) \times M E R_{h}\left(R_{i}\right)}
\end{aligned}
$$

In (3), $R_{i}$ denotes the $i$-th region among the mosaic candidate regions extracted in the previous step. $\operatorname{Num}\left(R_{i}\right)$ denotes the total number of pixels included in the candidate region $R_{i}$. $\operatorname{MER}_{h}\left(R_{i}\right)$ and $\operatorname{MER}_{v}\left(R_{i}\right)$ represent the length and width of the minimum enclosing rectangle for the candidate area of the mosaic, respectively.

First, the size feature of the candidate region is used to remove candidate regions of too small size generated by the influence of noise. The aspect ratio feature is used to effectively remove non-mosaic regions that are biased too long in either direction. Of course, there may be a mosaic area with a long elongated shape in a specific direction, but such an area is not found in actual harmful images. The density feature is used to remove low density non-mosaic regions in the region.

In this paper, the geometric features defined above are applied to the candidate regions of the mosaic. When the features fall below a predefined threshold[10], we judge them as nonmosaic regions and remove them from the candidate regions. In (3), $\mathrm{TH}_{\text {size }}, \mathrm{TH}_{\text {aspect }}$, and $\mathrm{TH}_{\text {compact }}$ denote the predefined thresholds for the size, aspect ratio, and density features of the candidate mosaic region, respectively. In this paper, these thresholds are artificially set through repetitive experiments.

In this paper, the three features defined above are applied to the candidate regions of the mosaic to remove all the regions determined to be non-mosaic regions, and then the remaining regions are finally determined as actual mosaic regions.

It is true that geometric features such as aspect ratio and size may remove common mosaic areas, for example, mosaic areas used to cover specific buildings or signs. However, in this paper, since the mosaic area to cover the body components is the main detection object, the geometric features used in this paper operate effectively. 


\section{Experimental results}

The personal computer used for the experiment in this paper consists of Intel Core (TM) i7 2.93 Ghz CPU and 8GB of main memory and used Microsoft's Windows 7 operating system. The proposed algorithm is implemented using Microsoft's Visual Studio and OpenCV open source computer vision library. In this paper, various indoor and outdoor input images including mosaic blocks were collected and used to compare and evaluate the performance of the proposed algorithm. These images are taken in various indoor and outdoor natural environments with no specific constraints set.

In this paper, we used an accuracy measure defined as in (4) and (5) to quantitatively evaluate the performance of the proposed mosaic detection algorithm [11]. $\mathrm{N}_{\mathrm{TP}}$ used in (13) and (14) indicates the number of mosaic regions accurately detected, $\mathrm{N}_{\mathrm{FP}}$ means the number of regions that are incorrectly detected as mosaic regions but not mosaic regions, and $\mathrm{N}_{\mathrm{FN}}$ denotes the number of mosaic regions not detected. In (13) and (14), $R_{\text {precision }}$ represents a relative proportion of mosaic regions accurately detected among all mosaic regions detected from the input image, and $\mathrm{R}_{\text {recall }}$ represents a relative ratio of mosaic regions accurately detected among all mosaic regions actually present in the image.

$$
\begin{aligned}
& R_{\text {precision }}=\frac{N_{T P}}{N_{T P}+N_{F P}} \\
& R_{\text {recall }}=\frac{N_{T P}}{N_{T P}+N_{F N}}
\end{aligned}
$$

In this paper, the performance of the proposed method is compared and evaluated in terms of accuracy with that of the existing template matching method. Since the proposed feature projection-based method reduces false detection of mosaic regions, it can be confirmed that it detects mosaic regions from images more robustly.

The proposed algorithm uses horizontal and vertical edge projection to detect the grid mosaic areas more accurately than the conventional method. However, when a mosaic is generated in a portion where image quality deterioration occurs in an input image, the detection accuracy of the proposed method may be slightly degraded. In this paper, line edge is basically used to reduce error caused by noise edge. Therefore, noise line edges below a certain size are not considered automatically, but errors due to noise line edges above that size are inevitable. To solve this problem, pre-processing such as image smoothing should be performed to reduce noise as much as possible, or the size of threshold, which determines a line edge, must be adaptively adjusted according to the degree of noise included.

The mosaic detected in this paper targets harmful images as described above. That is, it is difficult to include them in the paper because the experimental images are the ones in which the main parts of the body are exposed, or the images of the sexes of men and women are mostly taken. Therefore, in this paper, we select images that do not contain these parts and insert them into the paper.

\section{Conclusion}

In this paper, we propose a robust algorithm to detect the grid-type mosaic area from the input image based on horizontal and vertical line edge projection. In the proposed method, we first extract the Canny edge from the color image, and then extract the line edge connected continuously in the horizontal and vertical directions. The edges were projected in the horizontal and vertical directions, and the frequency of the projected edges was calculated to 
detect candidate regions of the mosaic. The detected candidate regions were then filtered using geometric features. Therefore, the non-mosaic regions were effectively removed from the candidate regions of the mosaic, and only the actual mosaic regions were accurately extracted.

In the future, we plan to increase the efficiency of the current mosaic detection algorithm by adaptively tuning various parameters used. In addition, the robustness of the algorithm will be improved by applying the images captured in various indoor and outdoor environments to the proposed algorithm.

\section{Acknowledgements}

This work was supported by the National Research Foundation of Korea (NRF) grant funded by the Korea government (MSIT) (2019R1F1A1056475).

\section{References}

[1] L. Yin, Q. Cheng, Z. Wang, and Z. Shao, Big Data for Pedestrian Volume: Exploring the Use of Google Street View Images for Pedestrian Counts. Appl. Geogr. 63 (2015)

[2] D. Guo, J. Tang, Y. Cui, J. Ding, and C. Zhao, Saliency-based Content-Aware Lifestyle Image Mosaics. J. Vis. Comm. Image Represent. 26 (2015)

[3] S.-W. Jang, M. Jung, Detection of Harmful Content Using Multilevel Verification in Visual Sensor Data. Wireless Pers. Comm. 86 (2016)

[4] X. Huang, H. Ma, and H. Yuan, Video Mosaic Block Detection Based on Template Matching and SVM. Proceedings of the IEEE International Conference for Young Computer Scientist, (2008) November 18-21; Hunan, China.

[5] Y.-J. Park, J.-W. Lee, G.-S. Choi, and J.-J. Park, A Study on Grid Mosaic Detection for Identifying Image Harmfulness. Proceedings of the Korea Society of Industrial Information Systems, (2015), July 19-20; Incheon, Korea.

[6] J. Liu, L. Huang, and J. Lin, An Image Mosaic Block Detection Method Based on Fuzzy C-Means Clustering. Proceedings of the IEEE International Conference on Computer Research and Development, (2011) March 1113; Shanghai, China. DOI: 10.1109/ICCRD.2011.5764011

[7] Z. Wei, J. Lin, L. Zhang, and S. Song, Mosaic Defect Detection Based on Macro Block Solid Edge Detection. Res. J. Appl. Sci. Eng. Tech. 5 (2013)

[8] S.-F. Sun, S.-H. Han, G. Wang, Y.-C. Xu, and B.-J. Lei, Mosaic Defect Detection in Digital Video. Proceedings of the IEEE Chinese Conference on Pattern Recognition, (2011) October 21-23; Chongqing, China.

[9] K. Gaurav and U. Ghanekar, Image Steganography Based on Canny Edge Detection, Dilation Operator and Hybrid Coding. Journal of Information Security and Applications 41 (2018) DOI: 10.1016/j.jisa.2018.05.001

[10] L. Jin, W. Zhang, G. Ma, E. Song, Learning Deep CNNs for Impulse Noise Removal in Images. J. Vis. Comm. Image Represent. 62 (2019)

[11] M. A. Elaziz, S. Lu, Many-Objectives Multilevel Thresholding Image Segmentation Using Knee Evolutionary Algorithm. Expert Syst. Appl. 125 (2019) 


\section{Authors}

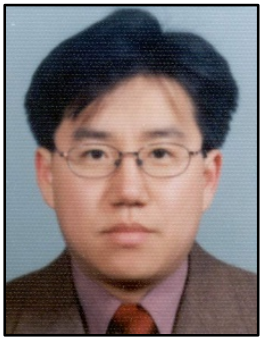

Seok-Woo Jang received the B.S., M.S., Ph.D. degrees in Computer Science from Soongsil University, Seoul, Korea, in 1995, 1997, and 2000, respectively. From October 2003 to January 2009, he was a Senior Researcher with the Construction Information Research Department at KICT, Korea. Since March 2009, he has been a Professor in the Department of Software, Anyang University, Korea. His primary research interests include robot vision, video indexing and retrieval, biometrics and pattern recognition.

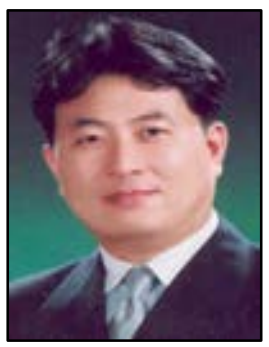

Sung-Youn Cho received his B.S. and M.S. degrees in Electronic Engineering from Hanyang University, Seoul, Korea, in 1987 and 1989, respectively. He received his Ph.D. degree in Computer Engineering from University of Wales, England in 1998. He is currently a professor in the Department of Software at Anyang University, Korea. His research focuses on image processing, pattern recognition, artificial intelligence, and situational awareness system. 